\title{
Delay Constrained Linear Transmission of a Mixture of Gaussian Measurements over a Fading Channel
}

\author{
Onur Tan*, Deniz Gündüz ${ }^{\dagger}$, Jesús Gómez Vilardebó* \\ ${ }^{*}$ Centre Tecnològic de Telecomunicacions de Catalunya (CTTC), Barcelona, Spain. \\ ${ }^{\dagger}$ Department of Electrical and Electronic Engineering, Imperial College London, London, UK.
}

\begin{abstract}
Delay constrained linear transmission (LT) of a mixture of Gaussian measurements over an additive white Gaussian noise (AWGN) fading channel is considered. At each time slot (TS), the control center (CC) asks for the measurement of a particular system parameter from the sensor, which is capable of measuring multiple independent system parameters. The average mean-square error (MSE) distortion is studied for Gaussian parameters and a Gaussian fading channel under an average power constraint. The optimal LT scheme is characterized under a strict delay constraint, and a graphical interpretation for the power allocation strategy is presented. Then, two achievable LT strategies are proposed for general delay constraints. It is shown that the performance improves as the delay constraint is relaxed, and when the delay constraint is completely removed, both strategies achieve the optimal performance under certain matching conditions.
\end{abstract}

\section{INTRODUCTION}

Wireless sensors are deployed throughout intelligent networks, such as smart grids (SGs), to closely track sensitive system parameters, e.g., voltage, current magnitudes, active/reactive power values, as well as temperature and other physical parameters [1]. The measurements are delivered to a control center (CC) that manages and controls the network efficiently. As the networks evolve and get smarter, near realtime accurate reconstruction of the measurements in the $\mathrm{CC}$ becomes necessary for fast response to failures. For example, in conventional state estimation for the electricity grid, measurements are collected once every two to four seconds and the state is updated once every few minutes [2]. However, more frequent state measurements and estimations are required for modern SGs, which inevitably imposes strict delay constraints on the transmission of measurements. Thus, linear transmission (LT), rather than advanced compression and channel coding techniques, has recently attracted attention for state estimation in intelligent networks, since LT reduces the delay and encoding complexity significantly; and additionally, limits the cost and energy requirements of the sensors.

LT of Gaussian sources has been extensively studied in the literature. Goblick showed in [3] that zero delay LT of a Gaussian source over an additive white Gaussian noise (AWGN)

This work was supported by the Spanish Government under project TEC2013-44591-P (INTENSYV). channel achieves the optimal mean-square error (MSE) distortion. In [4], the optimal LT scheme that matches an $r$ dimensional Gaussian signal to a $k$-dimensional AWGN vector channel under an average power constraint is characterized. LT of a Gaussian source over a fading AWGN channel is studied in [5]. It is shown that the optimal LT performance can be achieved by symbol-by-symbol processing, and increasing the block length does not provide any improvement, as opposed to nonlinear coding schemes. In [6], LT of noisy vector state measurements over a fading AWGN channel is studied under diagonal and general observation matrices. LT of vector Gaussian sources over multi-antenna static and fading channels is studied in [7].

Here, we consider a wireless sensor node that collects measurements from a mixture of $J$ Gaussian parameters, and delivers them to the CC over an AWGN fading channel under a delay constraint. Discretizing time into time slots (TSs), each corresponding to one use of the channel from the sensor to the $\mathrm{CC}$, we assume that the $\mathrm{CC}$ asks for the measurement of a particular parameter from the sensor at each TS. The sensor takes one sample of the requested parameter at each TS. Different from the studied multi-dimensional Gaussian source models, e.g., [4], [8], the sensor does not have the measurements of all the $J$ Gaussian parameters at each TS, and instead, one measurement is taken from one of the $J$ parameters at each TS. Hence, the source can be defined as a mixture of Gaussians. We assume that a measurement taken in a TS needs to be delivered to the CC within $d$ TSs. Thereby, after each transmission, the $\mathrm{CC}$ estimates the measurement whose deadline is just about to expire, i.e., the measurement that was taken $d-1$ TSs ago. We assume that the channel is fast fading; that is, its state is independent and identically distributed (i.i.d.) over TSs, and both the encoder and the decoder know the instantaneous channel state. We also assume that the statistics of the measured parameters, the parameter requests and the channel are known. Our goal is to estimate each requested measurement at the $\mathrm{CC}$ within the delay constraint with the minimum average MSE distortion.

We first derive the optimal LT strategy under a strict delay constraint $(d=1)$, and show that the optimal power allocation can be interpreted as water-filling reflected on a reciprocal mirror. Then, building on our previous work [9] 
and exploiting the results of [4], we propose two achievable LT strategies for larger delay constraints. In both strategies, measurements are first collected and stored depending on a delay constraint, and then, are transmitted to the CC over multiple channel accesses within the delay constraint. The two strategies consider different measurement selection criterias, which are used to select the appropriate stored measurement at each channel access. We then derive the theoretical lower bound (TLB) on the achievable MSE distortion. We characterize the average distortion achievable by the proposed LT schemes and the TLB under various power and delay constraints. We show that the achievable average distortion diminishes as the delay constraint is relaxed if the sensor is capable of measuring more than one system parameter. We also show that the proposed LT strategies meet the TLB when the delay constraint is completely removed; and hence, achieve the optimal performance under certain matching conditions.

\section{SySTEM MOdEL}

We consider a CC that monitors the operation of a complex system through a wireless sensor (Fig. 1). The sensor is capable of measuring $J$ distinct system parameters. The $j$ th system parameter is a zero-mean Gaussian random variable (r.v.) with variance $\sigma_{j}^{2}$, i.e., $\mathcal{N}\left(0, \sigma_{j}^{2}\right)$, for $j \in[1: J]$, where $[1: J]$ denotes the set $\{1,2, \ldots, J\}$. These system parameters are independent from each other, and their realizations are i.i.d. over time. In order to monitor the network operation, the CC requests the measurement of one system parameter from the sensor at each TS. The requested system parameter at each TS is modelled by the r.v. $M \in[1: J]$, which is also i.i.d. over time, with probability distribution $\operatorname{Pr}\{M=m\}=p_{M}(m)$. Thereby, the source $S$ can be described as a mixture of $J$ distinct Gaussian parameters, where the source sample is drawn from one of the $J$ independent Gaussian distributions with probability $p_{M}(m)$ at each TS.

We assume that the CC imposes a maximum delay constraint $d \in \mathbb{Z}^{+}$on the measurements, such that measurement requested in a TS needs to be transmitted within the following $d$ TSs, as otherwise; it becomes stale. The collected sensor measurements are transmitted to the $\mathrm{CC}$ over a fading channel with zero-mean and unit variance AWGN using an average power $P$. The channel output at TS $i$ is given by $y_{i}=h_{i} x_{i}+z_{i}$, where $x_{i}$ is the channel input, $z_{i}$ is the additive noise with $Z \sim \mathcal{N}(0,1)$, and $h_{i}$ is the fading state of the channel. We consider a fast-fading channel model, and assume that the fading coefficient $H_{i} \in \mathbb{R}$ is modelled as a r.v. i.i.d. over time with probability distribution $p_{H}(h)$.

Let $\mathbf{m}_{i}^{l}=\left[m_{i}, m_{i+1}, \ldots, m_{l}\right]$ be the sequence of the requested parameters at TSs $[i: l]$ for $i \leq l$. The measurement sequence is defined similarly as $\mathbf{s}_{i}^{l}=\left[s_{i}, \ldots, s_{l}\right]$, where the $i$-th entry $s_{i}$ is the measured value of the requested parameter $m_{i}$ at TS $i$. Therefore, the sequence $\mathbf{s}_{i}^{l}$ has independent entries, where the $i$-th entry comes from a Gaussian distribution with variance $\sigma_{m_{i}}^{2}$. The channel state and the output sequences within TSs $[i: l]$ are similarly defined as $\mathbf{h}_{i}^{l}=\left[h_{i}, \ldots, h_{l}\right]$ and $\mathbf{y}_{i}^{l}=\left[y_{i}, \ldots, y_{l}\right]$, respectively. We assume that both

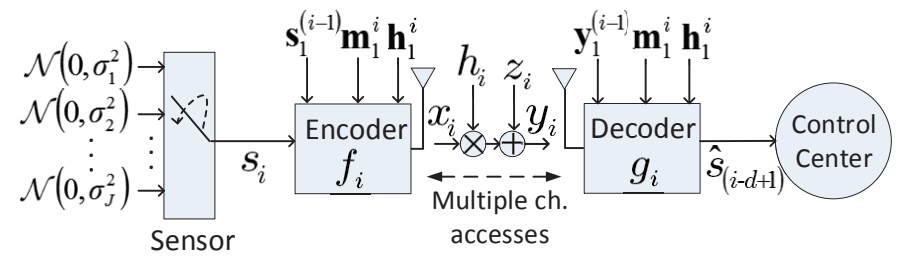

Fig. 1. The illustration of the transmission model from the perspective of the sensor with multiple channel accesses.

the encoder and the decoder know all the past accumulated channel states, $\mathbf{h}_{1}^{i}$, and requested parameters, $\mathbf{m}_{1}^{i}$, from the beginning to the current TS, as well as the statistics of the measured parameters, $\sigma_{m}^{2}$, the parameter requests, $p_{M}(m)$, and the channel, $p_{H}(h)$.

1) Encoding Function: The encoding function $f_{i}: \mathbb{R}^{i} \times$ $\mathbb{R}^{i} \times \mathbb{R}^{i} \rightarrow \mathbb{R}$, maps all the past measurements, $\mathbf{s}_{1}^{i}$, parameter requests, $\mathbf{m}_{1}^{i}$, and channel states, $\mathbf{h}_{1}^{i}$, to a channel input $x_{i}$ at each TS $i$, i.e., $x_{i}=f_{i}\left(\mathbf{s}_{1}^{i}, \mathbf{m}_{1}^{i}, \mathbf{h}_{1}^{i}\right)$. An average power constraint of $P$ is imposed on the encoding function:

$$
\bar{P} \triangleq \lim _{n \rightarrow \infty} \frac{1}{n} \sum_{i=1}^{n} \mathrm{E}_{M, H, S}\left[\left|X_{i}\right|^{2}\right] \leq P
$$

where the expectation is taken over $M, H$ and $S$.

2) Decoding Function: At the end of TS $i$, the goal of the CC is to estimate the measurement $s_{(i-d+1)}$, which has been requested exactly $d-1$ TSs ago, and is about to expire. The decoder for each TS $i$ observes all the past channel outputs, $\mathbf{y}_{1}^{i}$, parameter requests, $\mathbf{m}_{1}^{i}$, and channel states, $\mathbf{h}_{1}^{i}$, and reconstructs the measurement $s_{(i-d+1)}$ as $\hat{s}_{(i-d+1)}$. The decoding function at TS $i$ is denoted by $g_{i}: \mathbb{R}^{i} \times \mathbb{R}^{i} \times \mathbb{R}^{i} \rightarrow \mathbb{R}$, and we have $\hat{s}_{(i-d+1)}=g_{i}\left(\mathbf{y}_{1}^{i}, \mathbf{m}_{1}^{i}, \mathbf{h}_{1}^{i}\right)$. The average MSE distortion is given by:

$$
\bar{D} \triangleq \lim _{n \rightarrow \infty} \frac{1}{n} \sum_{i=d}^{n} \mathrm{E}_{M, H, S, Z}\left[\left|S_{(i-d+1)}-\hat{S}_{(i-d+1)}\right|^{2}\right],
$$

where the expectation is taken over $M, H, S$ and $Z$. In the scope of this paper, we are interested only in linear transmission policies in which $f_{i}$ are restricted to be linear functions of the sensor measurements. Under the linearity constraint on the encoding functions, $f_{i}$, the optimal estimators at the receiver, $g_{i}$, are also linear.

\section{Strict Delay Constraint}

We first consider a strict delay constraint $(d=1)$, that is, a measurement is transmitted and estimated within the current TS. In this case, the optimal LT performance is achieved by transmitting only the most recent measurement at each TS since all the previous measurements have expired, and transmitting expired measurement cannot help the estimation of the current measurement, since the previous measurements are independent of the current one. Therefore, since the channel input at TS $i$ depends only on the most recent measurement $s_{i}$, then the encoding function $f(h, m)$ is a scalar. Given the 


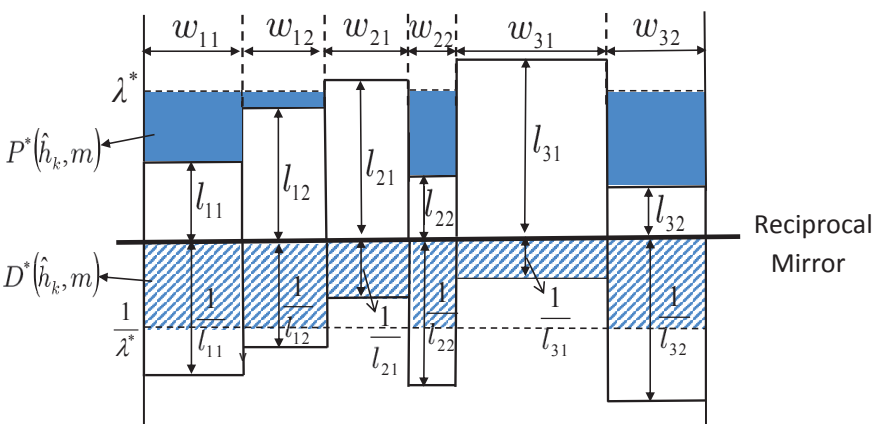

Fig. 2. Water-filling reflected on a reciprocal mirror.

encoding function, the decoding function that minimizes the MSE for Gaussian r.v.s is the linear MMSE estimator [10]. Then, the average MSE distortion $\bar{D}$, and the average power $\bar{P}$, as functions of $h$ and $\sigma_{m}^{2}$, can be written as:

$$
\begin{aligned}
\bar{D} & =\sum_{m=1}^{J} p_{M}(m) \int \frac{\sigma_{m}^{2}}{|h|^{2} f(h, m)^{2} \sigma_{m}^{2}+1} p_{H}(h) \mathrm{d} h, \\
\bar{P} & =\sum_{m=1}^{J} p_{M}(m) \int f(h, m)^{2} \sigma_{m}^{2} p_{H}(h) \mathrm{d} h .
\end{aligned}
$$

The optimal linear encoding function $f^{*}(h, m)$ is found as the solution to the convex optimization problem $\bar{D}^{*} \triangleq \min \bar{D}$, subject to the average power constraint $\bar{P} \leq P$, as follows :

$$
f^{*}(h, m)=\sqrt{\left[\frac{\lambda^{*}}{|h| \sigma_{m}}-\frac{1}{|h|^{2} \sigma_{m}^{2}}\right]^{+}},
$$

where $\lambda^{*}$ is the optimal Lagrange multiplier, such that $\bar{P}=P$.

The optimal power allocation function and the corresponding distortion are given by:

$$
\begin{aligned}
& P^{*}(h, m)=\frac{\sigma_{m}}{|h|}\left[\lambda^{*}-\frac{1}{|h| \sigma_{m}}\right]^{+}, \\
& D^{*}(h, m)=\frac{\sigma_{m}}{|h|} \min \left(\frac{1}{\lambda^{*}},|h| \sigma_{m}\right),
\end{aligned}
$$

where $\bar{D}^{*}=\mathrm{E}_{M, H}\left[D^{*}(h, m)\right]$ and $\bar{P}=\mathrm{E}_{M, H}\left[P^{*}(h, m)\right]$.

In Fig. 2, we present a graphical interpretation of the optimal power allocation and the corresponding distortion for $J=2$ parameters with variances $\sigma_{1}^{2}$ and $\sigma_{2}^{2}$, which are requested with probabilities $p_{M}(1), p_{M}(2)$, respectively. We also consider a discrete fading channel with three states, where the $k$ th state, $\hat{h}_{k}$, is observed with probability $p_{H}\left(\hat{h}_{k}\right), k=1,2,3$. Fig. 2 depicts rectangles that are placed upon a mirror surface and their reciprocally scaled images below. Rectangles represent all possible source-channel pairs $\left\{\sigma_{m}, \hat{h}_{k}\right\}$, where $l_{k m} \triangleq$ $\frac{1}{\left|\hat{h}_{k}\right| \sigma_{m}}$ and $w_{k m} \triangleq \frac{\sigma_{m}}{\left|\hat{h}_{k}\right|}$ indicate the height and width of the rectangles, respectively. The total power is poured above the level $l_{k m}$ up to the water level $\lambda^{*}$ across the rectangles placed above the mirror. The optimal power allocated to the sourcechannel pair $\left\{\sigma_{m}, \hat{h}_{k}\right\}$ is given by the shaded area below the water level and above $l_{k m}$. The corresponding distortion values are found by simply looking at the reciprocally scaled reflections of the rectangles and the water level on the mirror. If $\frac{1}{l_{k m}}>\frac{1}{\lambda^{*}}$, the distortion is given by the width $w_{k m}$ times the reciprocal of the water level $\frac{1}{\lambda^{*}}$, and if $\frac{1}{l_{k m}}<\frac{1}{\lambda^{*}}$, the distortion is $\sigma_{m}^{2}$, which are illustrated as dashed areas in Fig. 2. We call this as water-filling reflected on a reciprocal mirror.

\section{Achievable LT Strategies}

In this section, we propose two achievable LT strategies for general delay constraints $d \geq 1$. The block diagram of the proposed LT strategies is illustrated in Fig. 3. The LT strategies are composed of two main blocks, namely, storage and transmission blocks. There are two buffers of size $\bar{d}$ measurements, namely, the measurement buffer (MB) and the transmission buffer (TB). Here, we present these two schemes for an odd delay constraint $d \in\{1,3,5, \ldots\}$, but they can easily be adapted to the case when $d$ is even. In the storage block, given a delay constraint $d=2 \bar{d}-1$ for $\bar{d} \in[1: \infty]$, the sensor collects a block of $\bar{d}$ consecutive measurements at $\bar{d}$ consecutive TSs, and stores them into the MB. The consecutive blocks of $\bar{d}$ measurements, taken over successive time intervals, are indexed by $\bar{k}=1,2, \ldots$. Then, the $\bar{k}$-th block consists of the measurements taken within TSs $[(1+(\bar{k}-1) \bar{d}): \bar{k} \bar{d}]$, i.e., $\mathbf{s}_{(1+(\bar{k}-1) \bar{d})}^{\bar{k} \bar{d}}$. When the MB gets full with the $\bar{d}$ measurements of the $\bar{k}$-th block, the sensor removes $\mathbf{s}_{(1+(\bar{k}-1) \bar{d})}^{\bar{k}} \bar{d}$ from the $\mathrm{MB}$ and loads them into the TB. Then, for the next consecutive $\bar{d}$ TSs $[\bar{k} \bar{d}:((\bar{k}+1) \bar{d}-1)]$, the sensor accesses the channel and transmits a linear function of the measurements in the TB, i.e., $\mathbf{s}_{(1+(\bar{k}-1) \bar{d})}$, over the channel states $\mathbf{h}_{\bar{k} \bar{d}}^{((\bar{k}+1) \bar{d}-1)}$ satisfying the delay constraint $d$. The specifics of these linear functions will be explained below.

Note that, while the sensor transmits the measurements in the TB, it starts refilling the MB with new measurements $\mathbf{s}_{(\bar{k} \bar{d}+1)}^{(\bar{k} \bar{d}+\bar{d})}$. After $\bar{d}$ channel accesses within TSs $[\bar{k} \bar{d}:((\bar{k}+1) \bar{d}-$ $1)]$, the MB gets full again and its new $\bar{d}$ measurements are transferred to the TB for transmission over the next $\bar{d}$ TSs.

The proposed transmission strategies consist of two subblocks, namely, the measurement selection and scaling subblocks. This division is motivated by the results of [4], in which the ordered measurements are mapped one-to-one to the ordered channels and each measurement is transmitted over its corresponding channel. Hence, we assume that, at each channel access, the sensor selects only one measurement and scales it to a channel input value. However, in this case, we cannot directly use the optimal LT scheme in [4] and guarantee that the selected measurement and the channel state satisfy the optimal matching. This is because even though $\bar{d}$ measurements are available in the TB, states of the next $\bar{d}$ channels are not available to the transmitter as in the parallel channel model of [4]; and instead, they are becoming available over time through channel accesses. The two proposed LT strategies differ in the way they choose the measurement to be transmitted at each TS. 


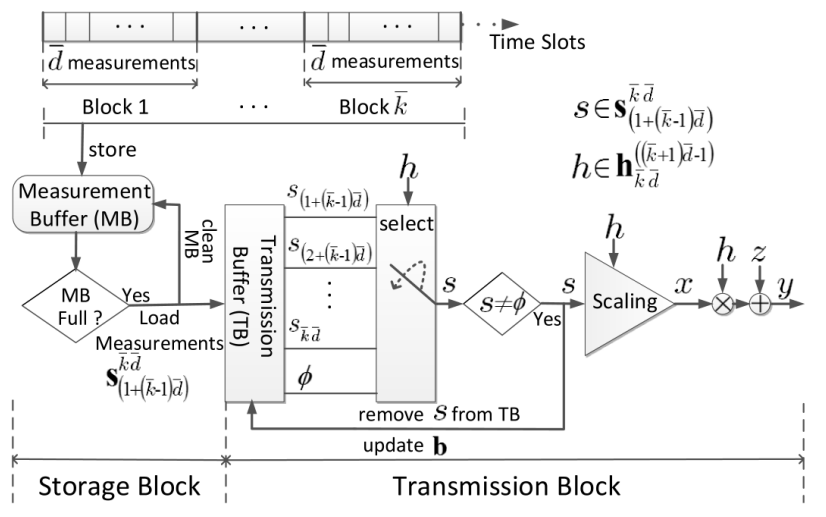

Fig. 3. The block diagram illustration of the proposed LT strategies.

\section{A. Linear Transmission Scheme with Hard Matching (LTHM)}

The first scheme we propose is called LT scheme with hard matching (LTHM), which has the following measurement selection criteria. Assume, without loss of generality, that the variances of the parameters are ordered in descending variances as $\sigma_{1}^{2}>\sigma_{2}^{2}>\cdots>\sigma_{J}^{2}$. We divide the magnitudes of channel states into $J$ ordered channel intervals, which are defined on the positive real line as, $\mathcal{H}_{m}=\left[H_{m}^{\prime}, H_{(m-1)}^{\prime}\right)$, where $H_{m}^{\prime}<H_{(m-1)}^{\prime}$ for $m \in[1: J]$. The boundary values are chosen as $H_{0}^{\prime}=\infty, H_{J}^{\prime}=0$ and $H_{m}^{\prime}=F_{H}^{-1}\left(1-\sum_{j=1}^{m} p_{M}(j)\right)$, for $m \in[1:(J-1)]$, where $F_{H}^{-1}()$ denotes the inverse of the cumulative distribution function of the channel magnitude $|h|$, $F_{H}(|h|)$.

Let $\mathbf{b}=\left[b_{1}, b_{2}, \ldots, b_{J}\right]$ be a $J$-length vector, where the $m$-th entry, $b_{m} \in[0: \bar{d}]$, denotes the number of measurements of parameter $m$ in the $\mathrm{TB}$, for $m \in[1: J]$. At each channel access, if $|h| \in \mathcal{H}_{m}$ and $b_{m} \neq 0$, then the sensor selects one measurement of the paramater type $m$ from the TB and feeds it to the scaling sub-block. If there are multiple measurements of the same parameter type $m$ in the TB, i.e., $b_{m}>1$, then the sensor selects one of them randomly. The selected measurement is removed from the TB and $\mathbf{b}$ is updated by reducing the $m$-th entry, $b_{m}$, by one. Thereby, each measurement is transmitted only once. On the other hand, if $|h| \in \mathcal{H}_{m}$ and $b_{m}=0$, no measurement is transmitted in that TS. Hence, LTHM considers a hard matching condition for selecting measurements, in which each parameter has a corresponding interval of channel states, and only measurements of that parameter can be transmitted over a channel state from that interval. Note that, since the channel state is known at the receiver, it also knows which type of measurement is transmitted at each TS.

For the scaling sub-block, we use the power allocation derived in Section III, which is also equivalent to the power allocation derived in [4]. Thus, the selected measurement of the parameter type $m$ is transmitted at the current channel state $|h| \in \mathcal{H}_{m}$, for $m \in[1: J]$, by allocating power $P(h, m)$, leading to distortion $D(h, m)$ :

$$
\begin{gathered}
P(h, m)= \begin{cases}{\left[\frac{\mu \sigma_{m}}{|h|}-\frac{1}{|h|^{2}}\right]^{+},} & \text {if hard matching holds, } \\
0, & \text { otherwise. }\end{cases} \\
D(h, m)= \begin{cases}\frac{\sigma_{m}^{2}}{|h|^{2} P(h, m)+1}, & \text { if hard matching holds, } \\
\sigma_{m}^{2}, & \text { otherwise, }\end{cases}
\end{gathered}
$$

where $\mu$ is chosen such that the average power constraint is satisfied.

After every transmission, the CC estimates the transmitted measurement $s$ by using the channel output $y$. It is noteworthy that after $\bar{d}$ channel accesses, we may have untransmitted measurements in the TB. TB is emptied anyway since these measurements have expired due to the delay constraint. These measurements are estimated with the maximum distortion $\sigma_{m}^{2}$. As we show next, the average number of untransmitted measurements decreases with the increasing delay constraint $d$. However, for a finite delay constraint, the untransmitted measurements dominate the distortion even for large average transmission power. In order to combat this drawback, we propose a different LT scheme which has a different measurement selection criteria that aims at adressing the limitation of LTHM.

\section{B. Linear Transmission Scheme with Soft Matching (LTSM)}

The LTSM retains the hard matching condition of LTHM, i.e., at each channel access, if $|h| \in \mathcal{H}_{m}$ and $b_{m} \neq 0$ for $m \in$ $[1: J]$, LTSM selects one measurement of the paramater type $m$ from the TB. Hence, LTSM also gives the highest selection priority to the measurement of the parameter type that satisfies the hard matching condition with the channel state. However, if $|h| \in \mathcal{H}_{m}$ and $b_{m}=0$, LTSM does not waste the channel state; and instead, it selects one measurement based on the following measurement selection criteria.

Assume that each interval $\mathcal{H}_{m}$ is further divided into two equally probable intervals by the boundary value $h_{m}^{\prime}=$ $F_{H}^{-1}\left(\frac{F_{H}\left(H_{(m-1)}^{\prime}\right)+F_{H}\left(H_{m}^{\prime}\right)}{2}\right)$, for $\forall m \in[1: J]$. If $|h| \in \mathcal{H}_{m}$ and $b_{m}=0$, then LTSM selects one measurement of parameter $\varsigma$, which is the parameter that minimizes the following distance metric:

$$
\min _{b_{\varsigma} \neq 0}|| h\left|-h_{\varsigma}^{\prime}\right| .
$$

When the hard matching condition is not satisfied, the LTSM considers a soft matching condition for selecting measurements; that is, among all parameter types of the measurements in the TB, it selects a measurement of the parameter whose corresponding interval of channel states has the value $h_{\varsigma}^{\prime}$ closest to the channel state magnitude $|h|$. If two distinct $\varsigma$ values satisfy the solution of Eqn. (8), then LTSM chooses the smallest value of $\varsigma$. LTSM allocates the power as in Eqn. (6), and transmits the selected measurement. At the end of $\bar{d}$ channel accesses, the sensor will have transmitted all the measurements in the TB, albeit some might have been allocated zero power as a result of the water-filling algorithm. 


\section{NO DELAY CONSTRAINT}

In this section, we first state the theoretical performance bound without any delay or complexity constraints. Then, we prove that this lower bound can be achieved by LTHM and LTSM under certain matching conditions and infinite delay.

\section{A. The Theoretical Lower Bound (TLB)}

Shannon's separation theorem states that the optimal endto-end distortion is achieved by concatenating the optimal source and channel codes when there is no delay or complexity constraints, and the source and channel distributions are ergodic [11]. The sensor can transmit to the $\mathrm{CC}$ at the ergodic capacity, $\bar{C}_{e}$, of the underlying fading channel, while the minimum distortion, $\bar{D}_{e}$, is found by evaluating the distortionrate function for a mixture of Gaussians source model at the ergodic channel capacity.

Since the channel state is known by both the transmitter and the receiver, the ergodic capacity, in terms of the optimal power allocation scheme $P_{e}^{*}(h)$, is given by $\bar{C}_{e} \triangleq$ $\mathrm{E}_{\mathrm{H}}\left[\frac{1}{2} \log \left(1+|h|^{2} P_{e}^{*}(h)\right)\right]$, where $P_{e}^{*}(h)$ is found by the water-filling algorithm as $P_{e}^{*}(h)=\left[\alpha^{*}-1 /|h|^{2}\right]^{+}$, such that $\alpha^{*}$ satisfies $\mathrm{E}_{\mathrm{H}}\left[P_{e}^{*}(h)\right]=P$.

The distortion-rate function of a mixture of $m$ Gaussian sources, $\mathcal{N}\left(0, \sigma_{m}^{2}\right)$, each of which is observed with probability $p_{M}(m)$ for $m \in[1: J]$, is defined as $\bar{D}_{e} \triangleq$ $\mathrm{E}_{\mathrm{M}}\left[\sigma_{m}^{2} 2^{-2 R_{e}^{*}\left(\sigma_{m}\right)}\right]$, where the rate allocated to source $m$, $R_{e}^{*}\left(\sigma_{m}\right)$, and the distortion of source $m, D_{e}^{*}\left(\sigma_{m}\right)$, are given by $R_{e}^{*}\left(\sigma_{m}\right)=\frac{1}{2}\left[\log \left(\frac{\sigma_{m}^{2}}{\beta^{*}}\right)\right]^{+}$and $D_{e}^{*}\left(\sigma_{m}\right)=\min \left(\beta^{*}, \sigma_{m}^{2}\right)$, respectively, such that $\beta^{*}$ satisfies $\mathrm{E}_{\mathrm{M}}\left[R_{e}^{*}\left(\sigma_{m}\right)\right]=\bar{C}_{e}$.

The achieved average distortion is $\bar{D}_{e} \triangleq \mathrm{E}_{\mathrm{M}}\left[D_{e}^{*}\left(\sigma_{m}\right)\right]$, which is the TLB on the achievable average distortion by any transmission strategy in our system model. Note that we have removed both the delay constraint and the linearity requirement on the encoder and decoder.

\section{B. Asymptotic Optimality of $L T$}

In general, TLB cannot be achieved by LT even if the delay constraint is removed. However, it can be shown that LTHM and LTSM meet the lower bound when the delay constraint is removed under certain matching conditions between the channel states and the parameter variances.

Assume that the channel follows a discrete fading distribution, where the channel state $h$ can take one of the $J$ values $\hat{h}_{m}$ with probability $p_{H}\left(\hat{h}_{m}\right)$ for $m \in[1: J]$. The discrete values are ordered as $\left|\hat{h}_{1}\right|>\left|\hat{h}_{2}\right|>\cdots>\left|\hat{h}_{J}\right|$. The next theorem states the necessary conditions in this discrete channel model under which LTHM and LTSM achieve the optimal distortion performance when the delay constraint is removed.

Theorem 1. For the discrete AWGN fading channel model, when the delay constraint is removed, i.e., $d \rightarrow \infty$, if the parameter variances and the discrete channel states satisfy $\frac{\sigma_{1}}{\left|\hat{h}_{1}\right|}=\cdots=\frac{\sigma_{J}}{\left|\hat{h}_{J}\right|}$, and $p_{M}(m)=p_{H}\left(\hat{h}_{m}\right)$, for $\forall m \in[1: J]$, then the TLB is achieved by LTHM and LTSM.

Proof. The proof is omitted here due to space limitations. It can be found in [12].

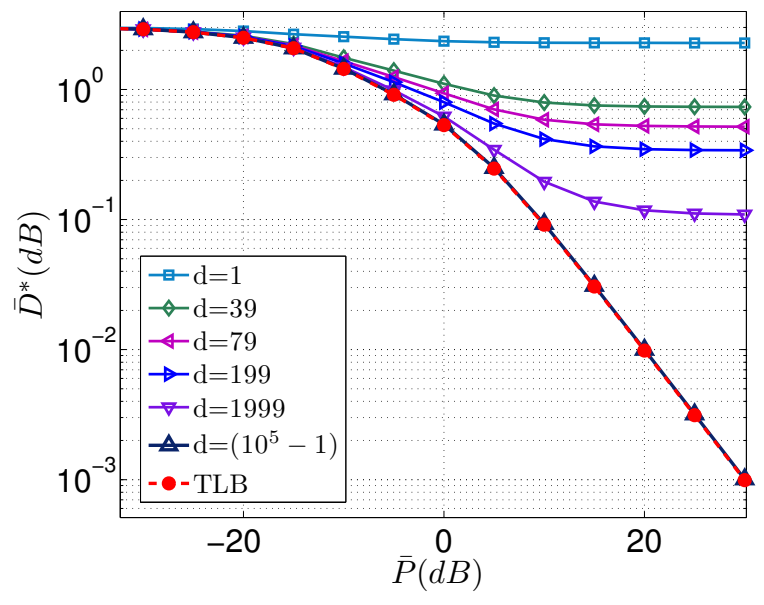

Fig. 4. The achievable average distortion of LTHM versus the average power for different delay constraints in the discrete fading channel model.

\section{NUMERICAL RESUltS AND OBSERVATIONS}

Here, we provide numerical results to compare the performances of LTHM and LTSM, and to analyze the impact of the delay and power constraints on the performance. In our simulations, we set $J=4$ Gaussian parameters with variances $\{10,5,1,0.5\}$, each of which is requested with probabilities $\{0.1,0.3,0.4,0.2\}$, respectively. For a continuous fading channel, we consider Rayleigh distribution with a scale parameter $\omega=3$, and for a discrete fading channel, we consider four states $\{\sqrt{10}, \sqrt{5}, 1, \sqrt{0.5}\}$, each of which is observed with probabilities $\{0.1,0.3,0.4,0.2\}$, respectively.

We investigate the achievable average distortion of LTHM versus average power for various delay constraints in the discrete channel setting in Fig. 4. We observe that the average distortion diminishes as the delay constraint is relaxed. This is because a relaxed delay constraint provides a larger number of measurements in the TB; and hence, more flexibility for the sensor in selecting the appropriate parameter measurement for each TS. We note that this statement does not hold when $J=1$, in which case increasing the block length for the LT of a Gaussian source does not provide any improvement on the performance [5]. As it can be seen in Fig. 4, the average distortion converges to a fixed value as the average power value increases. This is due to the effect of additional distortion brought in by the untransmitted measurements in the TB. The average number of untransmitted measurements and their effect on the average distortion decreases as the delay constraint is relaxed, since having a larger number of measurements in the TB increases the probability of finding a measurement that satisfies the hard matching condition. In particular, when the delay constraint is removed, as seen in Fig. 4, LTHM achieves the TLB, and becomes the optimal LT scheme, since the source-channel matching conditions in Theorem 1 are satisfied for the setup considered here.

Next, we illustrate in Fig. 5 the achievable average distortion of LTSM with respect to the average power under 


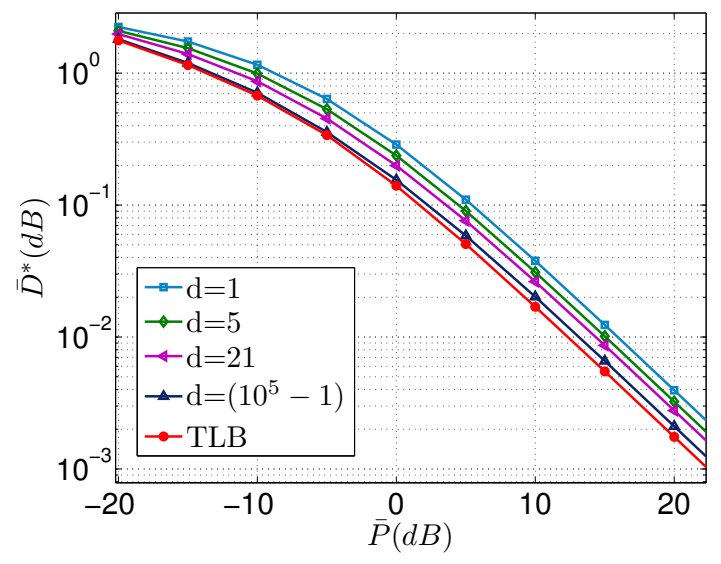

Fig. 5. The achievable average distortion of LTSM with respect to the average power for various delay constraints in the continuous fading channel model.

various delay constraints in the continuous channel model. Similarly to LTHM, the average distortion diminishes as the delay constraint increases. On the other hand, as opposed to LTHM, the average distortion achieved by LTSM decreases monotonically with the average power as illustrated in Fig. 5. This is because the performance of LTSM does not suffer from a fixed distortion component due to the untransmitted measurements. In addition, LTSM also approaches to the TLB as the delay constraint is relaxed. Although we do not expect the LTSM to meet the TLB in this setting since the matching conditions of Theorem 1 do not hold, we observe in Fig. 5 that it is very close to the TLB.

Finally, we compare the performances of LTHM and LTSM with each other and with the TLB. Fig. 6 shows the achievable average distortion of LTHM, LTSM and the TLB with respect to the delay constraint for the continuous fading channel and average power constraint $\bar{P}=10 \mathrm{~dB}$. As seen in the figure, the TLB is constant since it is derived by completely removing the delay and complexity constraints. As expected, the average distortion performances of LTHM and LTSM decrease as the delay constraint increases. For the setup considered in Fig. 6, LTSM outperforms LTHM for all delay constraints, while the gap between the two schemes decreases with the delay constraint. We expect that LTSM outperforms LTHM for all delay constraints. When the matching conditions of Theorem 1 hold, the two schemes both converge to the TLB.

\section{CONCLUSIONS}

We have studied the delay-constrained LT of mixture of Gaussian measurements from a sensor to a CC over an AWGN fading channel. We have considered a wireless sensor that can collect measurements from $J$ distinct Gaussian parameters. The CC asks for a measurement of a particular parameter from the sensor with a certain probability at each TS. In this framework, we have presented the optimal LT strategy under a strict delay constraint, and have given a graphical interpretation for the optimal power allocation scheme and

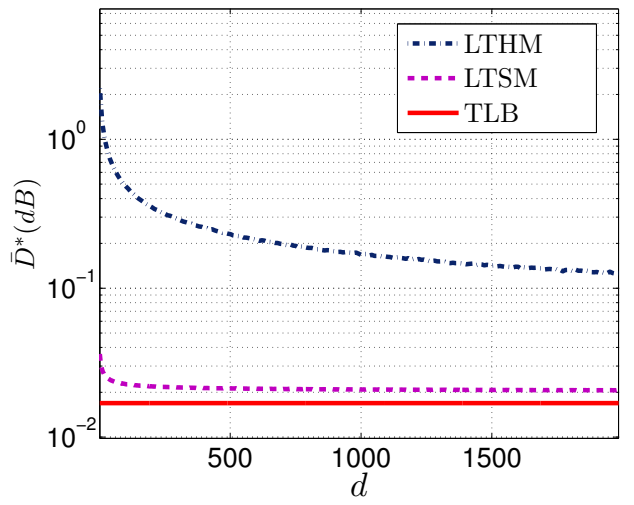

Fig. 6. The achievable average distortion of LTHM, LTSM and the TLB with respect to the delay constraint for the continuous fading channel model and average power constraint $\bar{P}=10 \mathrm{~dB}$.

the corresponding distortion value. We have proposed two achievable LT strategies for the transmission of the sensor measurements, and have provided numerical results to investigate the impact of the delay and average power constraints on their performances. We have seen that, if the number of parameters, $J$, is more than one, the average distortion decreases as the delay constraint is relaxed. We have derived a TLB on the achievable average distortion by relaxing the delay constraint and the linearity requirement. Our results have shown that the LTSM strategy performs better than LTHM for all delay constraints. We have also analytically shown that both strategies, under certain matching conditions between the parameter and the channel statistics, meet the TLB: and hence, are optimal, when the delay constraint is removed.

\section{REFERENCES}

[1] A. Ipakchi and F. Albuyeh, "Grid of the future," IEEE Power Energy Mag., vol. 7, no. 2, pp. 52-62, Mar.-Apr. 2009.

[2] A. Abur and A. G. Exposito, Power System State Estimation: Theory and Implemantation. New York, NY:CRC Press, 2000.

[3] T. Goblick, "Theoretical limitations on the transmission of data from analog sources," IEEE Trans. on Inf. Th., vol. 11, no. 4, pp. 558-567, Oct. 1965.

[4] K. Lee and D. Petersen, "Optimal linear coding for vector channels," IEEE Trans. Comm., vol. 24, no. 12, pp. 1283-1290, Dec. 1976.

[5] J. J. Xiao, Z. Q. Luo, and N. Jindal, "Linear joint source-channel coding for Gaussian sources through fading channels," in Proc. IEEE Global Telecommun. Conf., San Francisco, CA, Dec. 2006.

[6] J. Geng, H. Li, and L. Lai, "Smart grid system state measurement estimation over wireless channels," in Proc. 46th Inform. Sci. Sys. Conf., Princeton, NJ, Mar. 2012.

[7] I. E. Aguerri and D. Gündüz, "Linear transmission of correlated Gaussian sources over MIMO channels," in Proc. IEEE Int. Symp. on Wireless Commun. Systems (ISWCS), Ilmenau, Germany, Aug. 2013.

[8] A. Kashyap, T. Basar, and R. Srikant, "Minimum distortion transmission of Gaussian sources over fading channels," in Proc. IEEE Conf. Decision and Control, Maui, Hawai, Aug. 2013.

[9] O. Tan, D. Gündüz, and J. Gómez-Vilardebó, "Delay constrained linear transmission of smart grid state measurements," in Proc. IEEE Sensor Array Multich. Sig. Process. Wkshp., Coruña, Spain, Jun. 2014.

[10] A. E. Gamal and Y. H. Kim, Network Inf. Th. Cambridge University Press, 2011

[11] T. Cover and J. Thomas, Elements of Inf. Th. New York: Wiley, 1991.

[12] O. Tan, D. Gündüz, and J. Gómez-Vilardebó, "Linear transmission of a mixture of Gaussian measurements over a fading channel under delay constraints," - (in preparation). 